\title{
Design and Implementation of Gains Scheduled PI Control System for Shape Memory Alloy Actuator
}

\author{
Mohamed Alsayed Ahmed, Abdel Halim Bassiuny, and Elsayed Mokhtar Bakr
}

\begin{abstract}
Shape memory alloy (SMA) actuators provide unique features for motion control applications due to their compact design and high force to weight ratio. Integration of SMA actuators in motion control applications has not been widely employed due to their nonlinear behaviors and control difficulties. In this paper, the concept of model-based control of SMA actuators is introduced. Time variable parameter (TVP) model is used to describe the actuator dynamics. Gain scheduled Proportional-Integral (PI) controller with time variable gains has been developed based on pole placement technique. Experimental evaluation of gains schedule PI controller for SMA actuators shows stable and robust response that could compensate for the nonlinear phenomenon of these actuators.
\end{abstract}

Index Terms-Shape memory alloy actuators, nonlinear actuators, gains schedule controllers, tvp model.

\section{INTRODUCTION}

Shape memory alloys (SMAs) are a type of smart materials that can be deformed at low temperature and regains to its original undeformed configuration when heated to a higher temperature. SMAs possess interesting characteristics in terms of high power-to-weight ratio, large recovery strain, and low driving voltages. These characteristics make SMAs ideal for use as actuators. SMA actuators offer advantages over traditional actuators and have many potential applications such as robotic grippers [1], automotive mirror actuators [2], aerospace [3], etc.

However, SMA actuators have disadvantages, including slow speed and inaccurate response. The nonlinearity hysteresis effect that exists in SMA actuators has significant potential on the performance as it introduces delays and leads to inaccuracy in the control of these actuators [4].

Utilizing SMA actuators in real applications is, thus, constrained by the nonlinear behavior which requires complex control strategies [5]. In recent years, many control strategies ranging from simple controllers to highly sophisticated nonlinear controllers are applied to improve the performance of SMA actuators. Due to the nonlinear behavior with hysteresis, proportional controllers are generally inadequate for SMA actuators for critical applications. Mostly, the modified forms of classical controllers like PI, PD and PID are used for SMA actuators.

Sreekumar et al. [5] reviewed recent developments in nonlinear control technologies for SMA actuators. The nonlinear and hysteresis effects are formulated with the help

Manuscript received November 12, 2012; revised March 7, 2013.

The authors are with Mechanical Engineering Department, Helwan University (email: masterhelwan@yahoo.com, bassiuny@yahoo.com, mokhtarbakr@yahoo.com). of compensators and error governors [6]. Yee and Roy [7] presented architecture for force control using differential controller, which consists of a PID controller, a dynamic saturation block, and an anti-windup circuit for the integrator.

A PI type controller for radiant energy powered SMA actuator developed by Hull et al [8]. A halogen lamp was integrated to provide the radiant energy to activate SMA actuator. A modified PID controller called PID- $\mathrm{P}^{3}$ was proposed by Shameli et al. [9] for a typical SMA actuator, where a bending leaf spring was used to produce the bias force required to activate SMA actuator.

For larger values of error present in the position feedback, the cubic term of this controller produced great control effort that had considerable effects in reducing the settling time and overshoot of the system. For small error values, the cubic term vanishes and the controller works as a PID controller.

Most of these works required a precise system model, which makes the controller synthesis complicated and time consuming. Tai et al [4] investigated model predictive control algorithm as a method to compensate the SMA hysteresis phenomenon.

This paper focuses on force control of SMA actuator by integrating a gains schedule PI controller. Generally, Gains schedule for linear controllers are most effective for mapping the nonlinearity present in nonlinear control problems.

\section{EXPERIMENTAL SETUP}

Experimental apparatus was established to model the behavior of shape memory alloy (SMA) actuator also to online test the controller. The actuator consists of Flexinol ${ }^{\mathrm{TM}}$ wire from Dynalloy ${ }^{\circledR}[10]$ has the following specifications

- Diameter $=0.008$ inch , Length $=180 \mathrm{~mm}$

- Transition temperature $=90^{\circ} \mathrm{C}$

- Max operating current $=400 \mathrm{~mA}$

The experiment apparatus is concerned with modeling the force-current behavior, so that the wire is fixed from one terminal and the other terminal is fixed to a force sensor as shown Figure 1,2. The force sensor is a precise load cell from $\mathrm{NBC} \circledast$ (AH series $5 \mathrm{~kg}$ max capacity, 2 gram resolution). Load cell signal is amplified using NBC $®$ signal conditioner model SD03. The interface device is NI 6008 DAQ from National Instrument ${ }^{\circledR}$, which has 12-bit A/D converter and sampling rate of $10 \mathrm{kS} / \mathrm{s}$.

The experimental setup is used to collect the input/output data of SMA actuator to be modeled furthermore to apply the controller algorithm to the actuator hardware. The input current to SMA actuator is 0 to $400 \mathrm{~mA}$ current to induce internal wire force up to 1200 gram. SMA wire is preloaded with 295 gram force to prevent any slack when the current is disconnected. . The minimum force is the preload force with 
maximum 1200 gram force generated when the maximum current applied. Collected data is acquired using Labview ${ }^{\mathrm{TM}}$ program with $20 \mathrm{~Hz}$ sampling frequency and data set of 4047 samples.

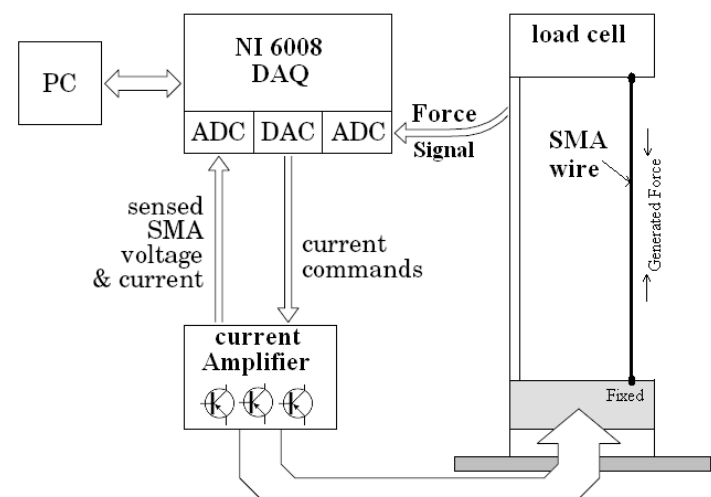

Fig. 1. Schematic diagram of SMA test rig

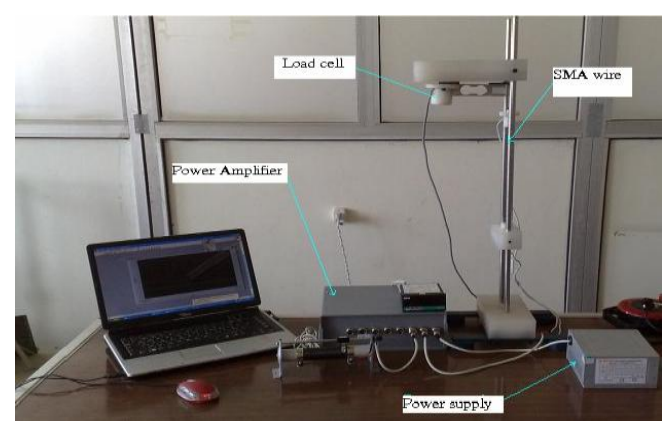

Fig. 2. SMA actuator test rig

\section{MODELING AND CONTROL}

\section{A. Linear Modeling and Identification}

Based on the experimental setup, Pseudo Random Signal (PRS) has been used to excite the system with different input current levels as shown in Figure. 3. Both input-output data are normalized by (1), (2) to remove any measurements offsets (i.e. preload force) which may affect the model estimation.

$$
\begin{aligned}
F_{\text {normalized }} & =\frac{F-F_{\min }}{F_{\max }-F_{\min }} \\
I_{\text {normalized }} & =\frac{I-I_{\min }}{I_{\max }-I_{\min }}
\end{aligned}
$$

Although, it is known that the dynamic behavior of SMA actuators is typically nonlinear but identification and estimation of a simple linearized model may be useful to determine the best regressors for the final nonlinear model structure. Therefore, as a first step, it is required to find a linearized representation of the dynamic system based on a transfer function (TF) model, with parameters identified and estimated from the measured data. The modeling approach is based on the discrete time transfer function on the following form (3).

where,

$$
y_{k}=\frac{B\left(z^{-1}\right)}{A\left(z^{-1}\right)} u_{k}
$$

$$
\begin{aligned}
& A\left(z^{-1}\right)=1+a_{1} z^{-1}+a_{2} z^{-2}+\cdots+a_{n} z^{-n} \\
& B\left(z^{-1}\right)=1+a_{1} z^{-1}+a_{2} z^{-2}+\cdots+a_{m} z^{-m}
\end{aligned}
$$

where $y_{k}$ is the output force, $u_{k}$ the input current, $a_{1} \ldots a_{n}$ and $b_{1} \ldots b_{m}$ are the model parameters, while $z^{-1}$. is the back ward shift operator, i.e. $z^{-1} y_{k}=y_{k-1}$. An appropriate structure for the transfer function (3) needs to be defined, i.e. $\{n, m$, $\delta$ where $\delta$ is the time delay, typically represented by setting $b_{1} \ldots b_{\delta-1}=0$. The two main statistical measures employed to help determine these values are the coefficient of determination $R_{T}^{2}$, which is a simple measure of the model fit (4).

where:

$$
\begin{gathered}
R_{T}^{2}=1-\frac{\operatorname{cov}\left(e_{k}\right)}{\operatorname{cov}\left(y_{k}\right)} \\
\operatorname{cov}\left(e_{k}\right)=\frac{1}{N} \sum_{k=1}^{N}\left(e_{k}-\bar{e}_{k}\right)^{2}, \\
\operatorname{cov}\left(y_{k}\right)=\frac{1}{N} \sum_{k=1}^{N}\left(y_{k}-\bar{y}_{k}\right)^{2}
\end{gathered}
$$

And, $e_{k}=y_{k}-\hat{y}_{k}, \bar{e}=\operatorname{mean}\left(e_{k}\right)$, and $\bar{y}_{k}=\operatorname{mean}\left(y_{k}\right)$ , $y_{k}$ is the actual measured output, an $\hat{y}_{k}$ is the theoretical response coming out from the model. The other statistical tool is Young Identification Criterion (YIC), which provides a combined measure of fit and parametric efficiency, with large negative values indicating a model which explains the output data well, without over-parameterization [11].

The present work utilizes the Simplified Refined Instrumental Variable (SRIV) algorithm to estimate the model parameters [11]-[12]. These statistical tools and associated estimation algorithms are apart from the Matlab® CAPTAIN toolbox [12]. The first step is to find the best identification structure. CAPTAIN $®$ toolbox was used to evaluate all models on the range $\{1,1,1\}$ to $\{3,3,3\}$. Table 1 shows the identification results which compose of the best ten linear models. The model ranking is based on both YIC and $R_{T}^{2}$. All ten models nearly have the same value of the coefficient of determination $R_{T}^{2}$ but they have a different YIC values. Since YIC for all models is negative, all ten models are controllable. The best two models have the following:

$$
\begin{gathered}
\mathrm{y}_{\mathrm{k}}=\frac{\mathrm{b}_{3} \mathrm{z}^{-3}}{1+\mathrm{a}_{1} \mathrm{z}^{-1}+\mathrm{a}_{2} \mathrm{z}^{-2}+\mathrm{a}_{3} \mathrm{z}^{-3}} \mathrm{u}_{\mathrm{k}} \\
y_{k}=\frac{b_{1} z^{-1}}{1+a_{1} z^{-1}} u_{k}
\end{gathered}
$$

The second model (6) has the least number of parameters; also it has a good YIC by comparing to the first model (1) which has a large number of parameters.

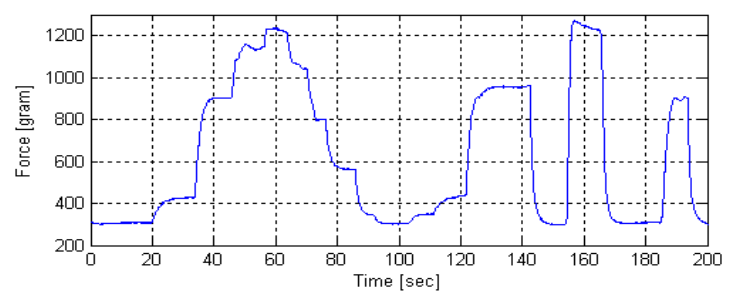




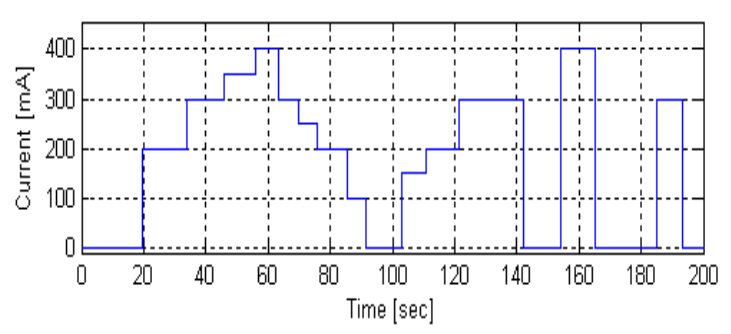

Fig. 3. SMA actuator output force for different input current levels

Identification criteria discussed above, reveal that a first order model with one numerator having one sample time delay provides the best estimated model and most optimum fit to $\{85 \%\}$ the data across a wide range of operating conditions. The parameters $a_{1}=-0.9656, b_{1}=0.0284$ yields the best overall fit.

\begin{tabular}{lccccc}
\hline \multicolumn{5}{c}{ TABLE I: THE BEST LINEAR MODELS } \\
\hline Rank & $\mathrm{n}$ & $\mathrm{m}$ & $\delta$ & $\mathrm{YIC}$ & $R_{T}^{2}$ \\
\hline 1 & 3 & 1 & 3 & -10.55 & 0.852 \\
\hline 3 & 1 & 1 & 1 & -9.02 & 0.850 \\
\hline 4 & 1 & 1 & 2 & -8.99 & 0.851 \\
\hline 5 & 1 & 1 & 3 & -8.96 & 0.851 \\
\hline 6 & 3 & 1 & 1 & -7.41 & 0.853 \\
\hline 7 & 2 & 1 & 1 & -6.21 & 0.852 \\
\hline 8 & 2 & 1 & 2 & -6.06 & 0.852 \\
\hline 9 & 2 & 1 & 3 & -5.89 & 0.853 \\
\hline 10 & 1 & 2 & 1 & -5.28 & 0.853 \\
\hline
\end{tabular}

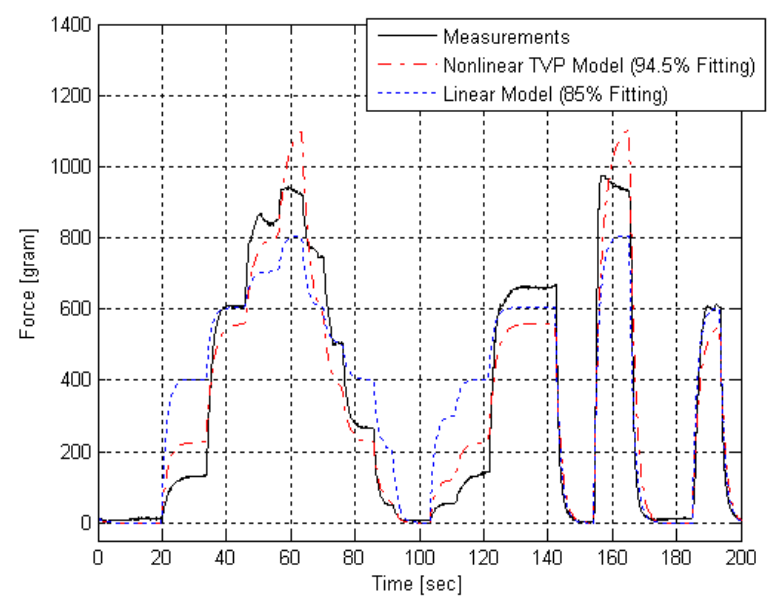

Fig. 4. Comparison of the best linear model and the best nonlinear TVP model for the data set

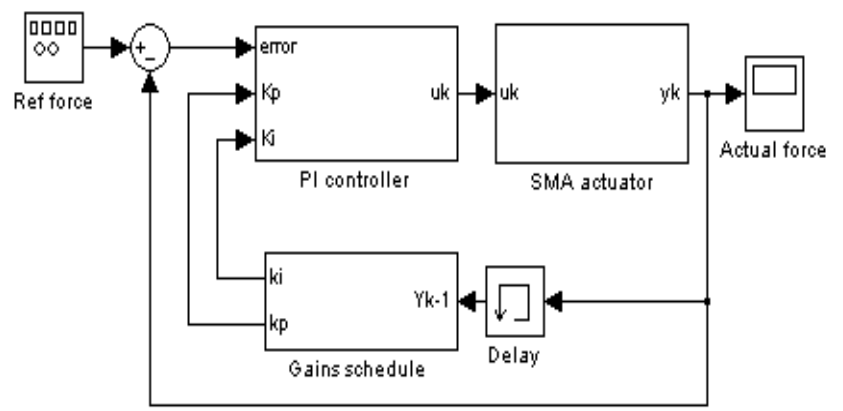

Fig. 5. Structure of Gains Scheduled PI controller

\section{B. Nonlinear Modeling and Identification}

The nonlinear modeling approach is based on the linear model with time variable parameter TVP. By rewriting the linear model (3) by TVP [12] form:

$$
\begin{aligned}
& y_{k}=\frac{B_{k}\left(\chi_{k}, z^{-1}\right)}{A_{k}\left(\chi_{k}, z^{-1}\right)} u_{k} \\
& A_{k}\left(\chi_{k}, z^{-1}\right)=1+a_{1}\left(\chi_{k}\right) z^{-1}+\cdots+a_{n}\left(\chi_{k}\right) z^{-n} \\
& B_{k}\left(\chi_{k}, z^{-1}\right)=b_{1}\left(\chi_{k}\right) z^{-1}+\cdots+b_{m}\left(\chi_{k}\right) z^{-m}
\end{aligned}
$$

where $A_{k}\left(\chi_{k}, z^{-1}\right)$ and $B_{k}\left(\chi_{k}, z^{-1}\right)$ are the time varying equivalents of $A\left(z^{-1}\right)$ and $B\left(z^{-1}\right)$. The notation indicates that the parameters are nonlinear functions of the vector $\chi_{k}$ where, in general, $\chi_{k}$ is defined in terms of any measured variables. By rewriting (6) to be in TVP form (8):

$$
y_{k}=\frac{b_{1}\left(\chi_{k}\right) z^{-1}}{1+a_{1}\left(\chi_{k}\right) z^{-1}} u_{k}
$$

The estimated value of $b_{1}$ is independent to the model input / output regressors, where $b_{1}=1.64 \times 10^{-4}$ By some trials and error, the best regressor for $a_{1}\left(\chi_{k}\right)$ is proportional to the square root of the output lag.

$$
\begin{gathered}
y_{k}=\frac{b_{1}\left(\chi_{k}\right) z^{-1}}{1+a_{1}\left(\chi_{k}\right) z^{-1}} u_{k} \\
a_{1}\left(\chi_{k}\right) \propto \sqrt{y_{k-1}}
\end{gathered}
$$

Using Captain toolbox and SRIV algorithm to estimate the actual fit for (10). Using first order polynomial fit on the form:

$$
a_{1}\left(y_{k}\right)=-3 \times 10^{-4} \sqrt{y_{k-1}}-0.9665
$$

Linear fitting for $a_{1}$ yields $R_{T}^{2}=0.945$ as shown in Fig. 4. For high order polynomial fitting, $R_{T}^{2}$ is insignificantly increased. The first order fitting is the most simple and efficient representation of the denominator coefficient $a_{1}$.

\section{Pole Placement Controller Tuning}

Gains schedule PI controller is based on the idea that, at each sampling instant, the TVP model (9) can be considered as a 'frozen' linear system. The control gains are determined at each sampling instant using pole placement linear methods. The general structure of linear discrete PI controllers may be presented by its closed loop characteristics equation as follows:

$$
D(z)=z^{2}+\left(a-1+k_{p} b+k_{I} b\right) z-\left(a+k_{p} b\right)
$$

By comparing (13) to the general second order equation of closed loop poles:

$$
\begin{aligned}
& \left(z+p_{1}\right)\left(z+p_{2}\right)=0 \\
& z^{2}+\left(p_{1}+p_{2}\right) z+p_{1} p_{2}=0
\end{aligned}
$$

These lead to

$$
p_{1} p_{2}=-\left(a_{1}+k_{p} b_{1}\right)
$$




$$
p_{1}+p_{2}=a_{1}-1+k_{p} b_{1}+k_{I} b_{1}
$$

The proportional gain is derived from the model by

$$
k_{p}=\frac{-p_{1} p_{2}-a_{1}}{b_{1}}
$$

Also the integral gain is derived from the model by

$$
k_{I}=\frac{p_{1}+p_{2}-a_{1}+1-k_{P} b_{1}}{b_{1}}
$$

By substituting the proportional gain equation (15) into (16)

$$
k_{I}=\frac{p_{1}+p_{2}+p_{1} p_{2}+1}{b_{1}}
$$

Controller gains are described in (15), (17) in terms of the model parameters $a_{1}$ and $b_{1}$. It is clear from (7) that the integral gain $k_{I}$ is independent to any regressor. On the other hand, the proportional gain $k_{p}$ is directly coupled to the denominator coefficient $a_{1}$ through $y_{k-1}$ regressor. Elimination of model parameters $a_{1}, b_{1}$ from the control gains expressions (15), (17) by substituting the model parameters in terms of the regressor $y_{k-1}$ from (9)

$$
\begin{array}{r}
k_{p}=\frac{-p_{1} p_{2}+3 \times 10^{-4} \sqrt{y_{k-1}}+0.9665}{1.64 \times 10^{-4}} \\
k_{I}=\frac{p_{1}+p_{2}+p_{1} p_{2}+1}{1.64 \times 10^{-4}}
\end{array}
$$

The stability conditions for (18), (19)

$$
\begin{aligned}
& k_{I}>0, k_{p}>0 \\
& \left|p_{1}\right|<1,\left|p_{2}\right|<1
\end{aligned}
$$

Closed loop poles pairs are assumed to be identical. The proportional gain $k_{p}$ increases as the poles approach the stability unit circle; furthermore, poles selection is restricted by the sampling time and the bandwidth of the ref trajectory. Practically, due to slow response of the SMA actuator the stable poles may lie on the range $\{0.6: 0.9\}$. These poles experimentally verified for $20 \mathrm{~Hz}$ sampling frequency. The complete structure of the controller is shown in Figure.5. The performance of the controller was experimentally investigated for different references inputs. Figures $6,7,8$ and 9 show the performance of the control system with respect to random references signal. The overshoot problem of linear PI controller is solved by the nonlinear version of gain scheduled PI controller. For sinusoidal and triangle trajectories, the gain schedule controller bandwidth less than the linear PI controller.

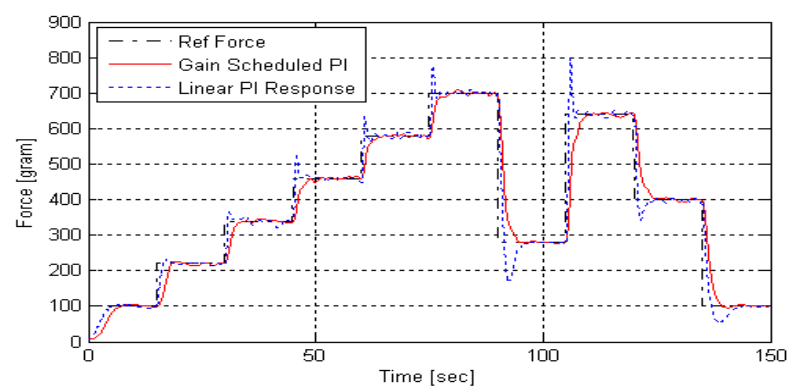

Fig. 6. Comparison of linear PI and gain scheduled PI controllers for step response

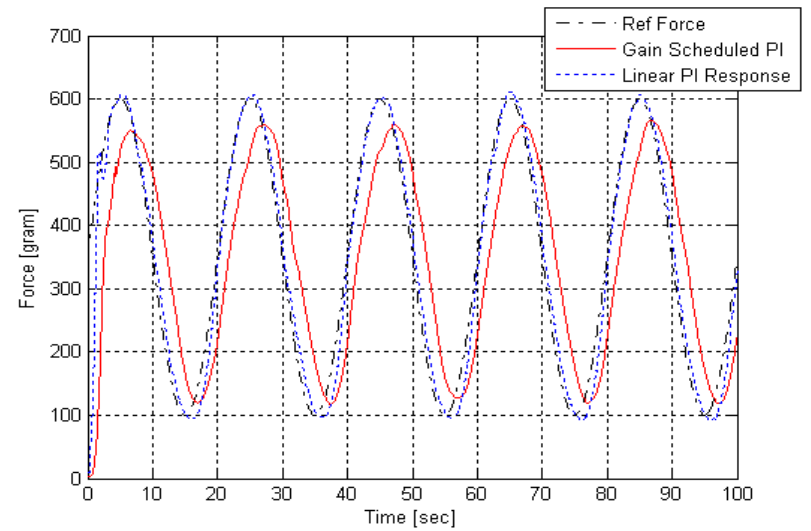

Fig. 7. Response of linear PI and gain scheduled PI controllers for $0.05 \mathrm{~Hz}$ sin ref trajectory

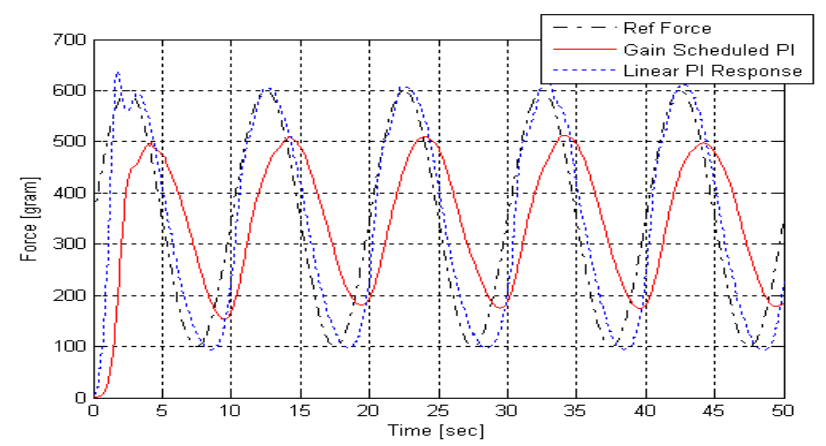

Fig. 8. Response of linear and gain scheduled PI controllers for $0.1 \mathrm{~Hz} \sin$ ref trajectory

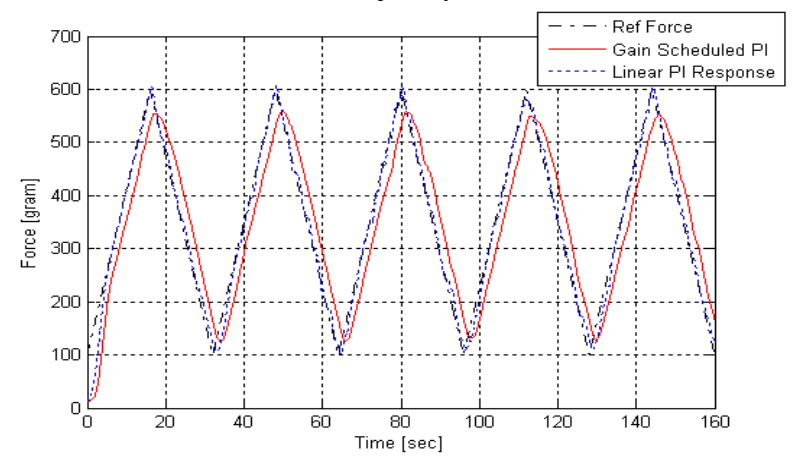

Fig. 9. Response of linear PI and gain scheduled PI controllers for triangle ref trajectory

\section{CONCLUSION}

In this paper an effective gains schedule PI controller for shape memory alloy actuator was developed. The controller structure is based on Time variable parameter TVP model which has state dependant gains. Due to the nonlinear dynamics of SMA actuators, the linear model cannot exactly simulate the actuator. TVP model is used to model such nonlinear dynamics. TVP model based on first order unit sample delay and two state dependent parameters is identified. State dependent parameters are continuously estimated every sampling instant based on the measured state. The best estimate of state for the SMA force actuator is based on the square root of the unit delay of the actuator output force). The success of using this state depends on the physical characteristics of SMA actuators. The output force is directly proportional to the square of the actuator current. Pole placement technique has been used to tune the controller by mapping the controller gains and the actuator measured 
output state. Experimental evaluation showed that the controller could achieve a good response to step and sinusoidal ref trajectories, and therefore could compensate for the hysteresis phenomenon of SMA actuators. The control system was able to satisfy a robust and stable performance which is suitable for low operating frequencies application.

\section{REFERENCES}

[1] K. Yang and Y. Wang, "Design, drive and control of a novel SMA-actuated humanoid flexible gripper," Journal of Mechanical Science and Technology, vol. 22, no. 5, pp. 895-904, 2008.

[2] E. Williams, G. Shaw, and M. Elahinia, "Control of an automotive shape memory alloy mirror actuator," Mechatronics, vol. 20, Issue 5, pp. 527-534, 2010

[3] M. Leester-Schadel. C. Hoxhold, and S. Demming, "Micro actuators on the basis of thin SMA foils," Microsystems Technol, vol. 14, pp. 697-704, 2008.

[4] N. T. Tai, N. B. Kha, and K. K. Ahn, "Predictive position and force control for shape memory alloy cylinders," Journal of Mechanical Science and Technology, vol. 24, no. 8, pp. 1717-1728, 2010.

[5] M. L. Sreekumar and Singaperumal, "Recent advances in nonlinear control technologies for shape memory alloy actuators," Journal of Zhejiang University SCIENCE A, 2007.

[6] M. L. Tharayil, A. G. Alleyne, "Modeling and control for smart mesoflap aero elastic control," IEEE/ASME Transactions on Mechatronics, vol. 9, no. 1, pp. 30-39, 2004.

[7] H. T. Yee and F. Roy, "Architecture for Fast and Accurate Control of Shape Memory Alloy Actuators," International Journal of Robotic. Research, vol. 27, no. 5, May 2008.

[8] P. V. Hull, S. L. Canfield, and C. Carrington, "A radiant energy-powered shape memory alloy actuator," Mechatronics, vol. 14 no. 7, pp. 757-775, 2004.

[9] E. Shameli, A. Alasty, and H. Salaarieh, "Stability analysis and nonlinear control of a miniature shape memory alloy actuator for precise applications," Mechatronics, 2004.

[10] FlexinolTM datasheet. [Online]. Available: http://www.dynalloy.com.

[11] P. C. Young, "Simplified Refined Instrumental Variable (SRIV) Estimation and True Digital Control (TDC)," in Proc. a tutorial introduction, 1st Europeaan Control Conference, pp. 1295-1306 (Grenoble), 1991.
[12] P. C. Young, "Simplified Refined Instrumental Variable (SRIV) Estimation and True Digital Control (TDC)," in Proc. a tutorial introduction, 1st Europeaan Control Conference, pp. 1295-1306 (Grenoble), 1991.

[13] P. C. Young, "Data-Based Mechanistic Modelling of Engineering Systems," Journal of Vibration and Control, vol. 4, pp. 5-28, 1998.

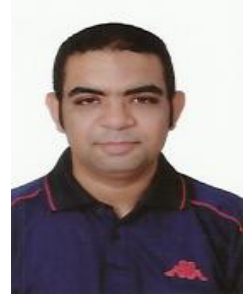

Mohamed Alsayed Ahmed was born in Giza-Egypt in $27^{\text {th }}$ of December 1984. He received the MS.C. degree in the field of Mechatronics Engineering from University of Helwan $17^{\text {th }}$ March 2012. He is currently a lecturer assistant in Mechanical Engineering Department, Faculty of Engineering, Helwan University. His researches are in the field of Robotics, Mechatronics, Smart Actuators and Solar Energy Applications.

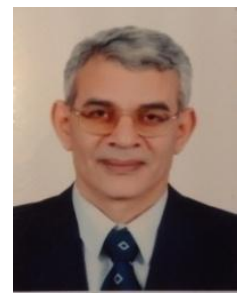

Abdel Halim Bassiuny was born in Abdel Halim Bassiuny, Cairo-Egypt in $23^{\text {rd }}$ of October 1955 . He received the $\mathrm{Ph} . \mathrm{D}$. degree in the field of computer process control in 1990 according to the channel program between Helwan Uni. (Egypt) and Wuppertal Uni. (Germany). Since 1990 he has been an assistant professor and he is currently associate professor in the Department of Mechanical Engineering, Helwan University. His research interests include systems control theory and its applications, mechatronic systems and intelligent condition monitoring systems.

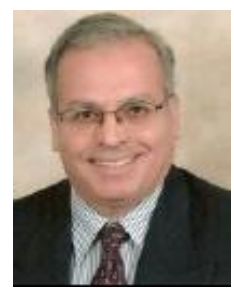

Elsayed Mokhtar Bakr was born in Cairo-Egypt in $30^{\text {th }}$ of August 1952. He received the Ph.D. degree in the field of flexible Multibody System Dynamics from University of Illinois (UIC), U.S.A, and $22^{\text {nd }}$ March 1987. He is currently a full Professor in Mechanical Engineering Department, Faculty of Engineering, Helwan University. Most of his researches are in the field of Medical Robotics, Multibody System Dynamics, Kinematics and Dynamics of Flexible Robot Manipulators, Mechanics of Legged Locomotion Systems and Nonlinear Finite Element Analysis. 